\title{
AN EXACT SOLUTION FOR STEADY STATE MAGNETIC RECONNECTION IN THREE DIMENSIONS
}

\author{
I. J. D. Craig, R. B. Fabling, S. M. Henton, and G. J. Rickard \\ University of Waikato, Hamilton, New Zealand \\ Received 1995 May 9; accepted 1995 October 11
}

\begin{abstract}
An exact three-dimensional solution is derived for the steady state magnetic reconnection of incompressible, resistive plasmas. The analysis provides a natural extension of the analytic, two-dimensional reconnection solution of Craig \& Henton. The solution shows how advective motions through the separatrix "spine-curve" lead to global current sheets aligned to the separatrix "fan."
\end{abstract}

Subject headings: MHD — plasmas

\section{INTRODUCTION}

In a recent paper, Craig \& Henton (1995) constructed an exact analytic solution for steady state, incompressible magnetic reconnection in two-dimensional planar geometries. The solution includes previous magnetic-annihilation modelswhich are restricted to the merging of antiparallel field lines - in the limiting case of vanishing shear flows across the neutral point (see, e.g., Phan \& Sonnerup 1990; Besser, Biernat, \& Rijnbeek 1991; Jardine et al. 1992). However, when the conventional stagnation-point flow symmetries are relaxed to include shear, curved field lines can be rapidly reconnected across an X-type neutral point. The reconnection rate is independent of the plasma resistivity, yet the bulk of the magnetic energy is released as ohmic heating as opposed to the kinetic energy of mass motion.

The purpose of this Letter is to indicate the extension of the Craig-Henton solution to three-dimensional geometries. The properties of the reconnective system are outlined in $\S 2$, where we construct the general three-dimensional solution. A discussion of the two- and three-dimensional reconnection topology is given in $\S 3$. Our findings are summarized in $\S 4$.

\section{THE STEADY STATE SOLUTION}

\subsection{The Reconnective System}

The reconnective system is governed by the steady state momentum and induction equations for an incompressible, resistive plasma. The system can be written in the nondimensional form

$$
\begin{gathered}
(\boldsymbol{v} \cdot \boldsymbol{\nabla}) \boldsymbol{\omega}-(\boldsymbol{\omega} \cdot \boldsymbol{\nabla}) \boldsymbol{v}=(\boldsymbol{B} \cdot \boldsymbol{\nabla}) \boldsymbol{J}-(\boldsymbol{J} \cdot \boldsymbol{\nabla}) \boldsymbol{B}, \\
(\boldsymbol{v} \cdot \boldsymbol{\nabla}) \boldsymbol{B}-(\boldsymbol{B} \cdot \boldsymbol{\nabla}) \boldsymbol{v}=\eta \boldsymbol{\nabla}^{2} \boldsymbol{B},
\end{gathered}
$$

where the magnetic and velocity fields satisfy

$$
\boldsymbol{\nabla} \cdot \boldsymbol{B}=0, \quad \boldsymbol{\nabla} \cdot \boldsymbol{v}=0
$$

and the current density and fluid vorticity are given by

$$
\boldsymbol{J}=\boldsymbol{\nabla} \times \boldsymbol{B}, \quad \boldsymbol{\omega}=\boldsymbol{\nabla} \times \boldsymbol{v} .
$$

It is clear that energy losses from the system can occur only through the ohmic heating of the plasma. Since the dimensionless resistivity $\eta$ is typically very small, of order $10^{-10}$, resistive effects are significant only in localized regions of high current density. It is the finite resistivity that allows topological change in the global magnetic field via magnetic reconnection, a process involving the cutting and rejoining of field lines at null points of the field.

\subsection{Preliminary Observations}

We note that the symmetry in the $\boldsymbol{B}$ - and $\boldsymbol{v}$-fields is broken only by the resistive term. It follows that simple potential field solutions exist in which irrotational flows $(\boldsymbol{\omega}=\mathbf{0})$, supported by gradients in the plasma pressure, are constrained to potential field lines.

Another special case occurs when the field lines are straight. Consider, for instance, the simple one-dimensional field $\boldsymbol{B}=Y(x) \hat{\boldsymbol{y}}$. The right-hand side of the momentum equation vanishes, so any velocity field that reduces the left-hand side to zero-such as a potential flow-provides a possible solution. This construction is central to magnetic-annihilation models in which straight field lines are advected into a global current sheet layer by stagnation-point flows (Sonnerup \& Priest 1975).

\subsection{Reconnective Solutions}

We now consider solutions in which one-dimensional fields are superposed onto "background" potential field solutions. From Craig \& Henton (1995), we know that the threedimensional stagnation-point field, namely

$$
\boldsymbol{P}=\alpha[-x \hat{\boldsymbol{x}}+\kappa y \hat{\boldsymbol{y}}+(1-\kappa) z \hat{\boldsymbol{z}}],
$$

where $\alpha$ and $\kappa$ are constant, is the only admissible potential field. It is sufficient therefore to consider the forms

$$
\begin{gathered}
\boldsymbol{B}=\lambda \boldsymbol{P}+\boldsymbol{Q}(x), \quad \boldsymbol{Q}=Y(x) \hat{\boldsymbol{y}}+Z(x) \hat{\boldsymbol{z}}, \\
\boldsymbol{v}=\boldsymbol{P}+\boldsymbol{q}(x), \quad \boldsymbol{q}=v(x) \hat{\boldsymbol{y}}+w(x) \hat{z},
\end{gathered}
$$

where $\lambda$ is some constant.

Substituting these forms into equations (1) and (2) gives the solution

$$
v(x)=\lambda Y(x)+\gamma_{1} x, \quad w(x)=\lambda Z(x)+\gamma_{2} x,
$$

where $Y(x)$ and $Z(x)$ satisfy respectively the differential equations

$$
\begin{array}{r}
\alpha\left(1-\lambda^{2}\right)\left(\kappa Y+x Y^{\prime}\right)+\eta Y^{\prime \prime}=\alpha \gamma_{1} \lambda(\kappa+1) x, \\
\alpha\left(1-\lambda^{2}\right)\left[(1-\kappa) Z+x Z^{\prime}\right]+\eta Z^{\prime \prime}=\alpha \gamma_{2} \lambda(2-\kappa) x,
\end{array}
$$

subject to the conditions

$$
\gamma_{2} \kappa=\gamma_{1}(1-\kappa)=0 .
$$


The solution comprises five internal parameters, $\alpha, \kappa, \lambda, \gamma_{1}$, and $\gamma_{2}$, but only three can be chosen independently in any concrete application. Obviously, $\alpha$ and $\kappa$ determine the magnitude and direction of $\boldsymbol{P}$. A three-dimensional solution requires $0<\kappa<1$, and the choice $\kappa=\frac{1}{2}$ corresponds to axisymmetry. No flow ever crosses the plane $x=0$, but for positive $\alpha$ there is material inflow through all other planes $|x|>0$. The solution degenerates to planar by taking either $\kappa=0$ or $\kappa=1$; in such cases $\gamma_{1}$ or $\gamma_{2}$ can be chosen to be nonzero.

More significant, in terms of magnetic reconnection, is the role of $\lambda$. For $\lambda>0$ the stagnation-point symmetry is lost, shear flows develop, and curved field lines reconnect across the neutral point.

\section{INTERPRETATION OF THE SOLUTION}

\subsection{Two-dimensional Solutions}

Before discussing the fully three-dimensional analysis we outline the simpler planar reconnection solutions. Suppose $z$ is the ignorable coordinate. Then we must take $\kappa=1$ and specify $\alpha, \lambda$, and $\gamma_{1}$. In the case $\gamma_{1}=0$ we can integrate equations (9) and (10) to yield

$$
\boldsymbol{v}=\boldsymbol{P}+\lambda \boldsymbol{Q}(x), \quad \boldsymbol{B}=\lambda \boldsymbol{P}+\boldsymbol{Q}(x),
$$

where

$$
\begin{gathered}
\boldsymbol{Q}(x)=\frac{E}{\eta \mu} \text { daw }(\mu x) \hat{\boldsymbol{y}}+\left[\frac{\sqrt{\pi}}{2 \mu} Z^{\prime}(0) \operatorname{erf}(\mu x)+Z(0)\right] \hat{z}, \\
\text { daw } x=\int_{0}^{x} \exp \left(t^{2}-x^{2}\right) d t, \quad \mu^{2}=\frac{\alpha}{2 \eta}\left(1-\lambda^{2}\right),
\end{gathered}
$$

and $E$ is a constant of integration.

The planar reconnection solution of Craig \& Henton (1995) is obtained on setting $Z(0)=Z^{\prime}(0)=0$. A global current sheet of amplitude $O\left(\eta^{-1}\right)$ is centered on the plane $x=0$. Strong shearing motions are associated with the reconnection region close to the neutral point. The shear layer vanishes in the special case $\lambda=0$, and the solution reduces to the annihilation of straight field lines advected by stagnation-point flow $\boldsymbol{v}=\boldsymbol{P}=\alpha(-x \hat{\boldsymbol{x}}+y \hat{\boldsymbol{y}})$. However, in all cases the width of the current layer is determined by the behavior of the Dawson function: specifically, daw $(\mu x)$ is an odd function that peaks when $\mu x \approx 0.92$-i.e., when $x$ is $O(\sqrt{\eta})$-and declines as $1 / 2 \mu x$ for large arguments. It follows that the influence of the disturbance field $Y(x)$ rapidly diminishes outside the current layer.

Consider now the case $\boldsymbol{Q}=Z(x) \hat{z}$. If $|Z(0)|>0$, there is only a planar null, rather than a true null of the field. To determine the flux-annihilation rate we take $Z(0)=0$ and scale $Z^{\prime}(0) \sim \mu \sim \eta^{-1 / 2}$ to normalize the field amplitude $|Z|$ on the boundary plane $x=1$ (say). The rate is slow since $\eta J=\eta Z^{\prime}(0) \sim \eta^{1 / 2}$.

More generally, the pressure distribution required to sustain the flow is given by

$$
P(x, y)=P_{0}-\frac{1}{2}\left[u_{H}^{2}+Y(x)^{2}+Z(x)^{2}\right]-\alpha \lambda y Y(x),
$$

where $\boldsymbol{u}_{H}=(-\alpha x, \alpha y)$. The pressure is clearly dominated by the buildup in the planar field amplitude, $Y \sim \eta^{-1 / 2}$. Specifically, $P>0$ implies the scaling $P_{0} \sim \eta^{-1}$. Thus, in the outer field where $B=O(1)$, the plasma $\beta$ must be huge, $\beta=\frac{1}{2} P_{0} /$ $B^{2} \sim \eta^{-1}$, to sustain fast planar reconnection.

Finally, we mention the effect of taking $\gamma_{1}$ to be nonvanishing. As discussed by Craig \& Henton, a global shearing flow is superposed onto the intrinsic solution. In this case equation (12) provides a solution of the homogeneous problem.

\subsection{Three-dimensional Solutions}

The previous analysis shows that the three-dimensional solution has the generic form

$$
\boldsymbol{v}=\boldsymbol{P}(\boldsymbol{x})+\lambda \boldsymbol{Q}\left(\boldsymbol{x} \cdot \hat{\boldsymbol{x}}_{i}\right), \quad \boldsymbol{B}=\lambda \boldsymbol{P}(\boldsymbol{x})+\boldsymbol{Q}\left(\boldsymbol{x} \cdot \hat{\mathbf{x}}_{i}\right),
$$

where $\boldsymbol{x}=x_{i} \hat{\boldsymbol{x}}_{i}$. In our present representation with $\boldsymbol{Q}=\boldsymbol{Q}(x)$, there are three independent internal parameters, namely, $\alpha, \kappa$, and $\lambda$. Constants of integration arising from the solution of equations (9) and (10) can be regarded, in common with $E$ in equation (13), as external control parameters of order unity.

How does three-dimensional reconnection differ from the planar two-dimensional model? Perhaps the most significant change is the impact of the nonremovable null point on the separatrices of the field. Close to the origin, the field-line equations reduce to

$$
-\frac{d x}{x}=\frac{d y}{\kappa y+a_{1} x}=\frac{d z}{(1-\kappa) z+a_{2} x},
$$

where $a_{i}=E_{i} / \alpha \lambda \eta$ and the $E_{i}$ are integration constants (as in eq. [13]). These integrate to yield

$$
x^{\kappa}\left(y+\frac{a_{1}}{1+\kappa} x\right)=c_{1}, \quad x^{1-\kappa}\left(z+\frac{a_{2}}{2-\kappa} x\right)=c_{2}
$$

for the $y(x)$ and $z(x)$ dependencies. The separatrices, defined as the totality of field lines threading the neutral point, are determined by setting $c_{1}=c_{2}=0$. We obtain the plane $x=0$ plus the line $y=-a_{1} x /(1+\kappa), z=-a_{2} x /(2-\kappa)$. Following Priest \& Titov (1995), we call these the "fan" and "spine," respectively. Equation (17) now gives $z(y)=c_{3} y^{(1-\kappa) / \kappa}$ for the field lines on the fan.

Figure 1 details the fan and spine structure for a typical calculation. Figure 2 shows how curved field lines are advected



FIG. 1.-Spine curve plus separatrix fan in the plane of the current sheet $x=0$. The rays of the fan are straight because of axisymmetry. Parameters are $\alpha=2, \quad \kappa=\frac{1}{2}, \lambda=0.9, E_{1}=E_{2}=0.1$, and $\eta=0.05$. The neutral point is assumed to lie at the center of the cube $-2 \leq x, y, z \leq 2$. 


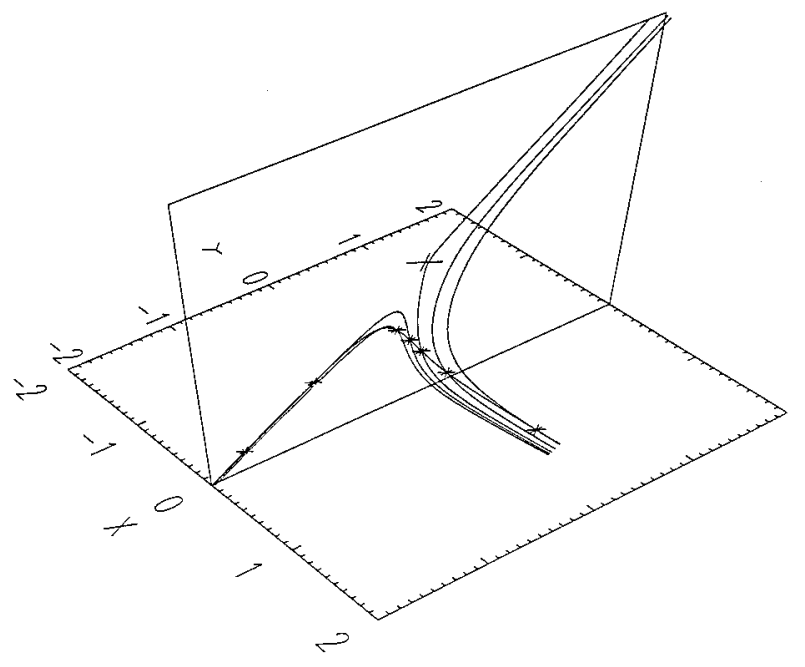

FIG. 2.-Diagram of a curved magnetic field line carried by a fluid element (asterisks) reconnecting at the neutral point (cross). Reconnection occurs as the particle passes through the spine curve before exiting along a ray in the plane of the current sheet. Solution parameters as in Fig. 1.

into the current plane $x=0$-across which there is no flow-by the motions of a fluid element. The field line reconnects at the neutral point as the fluid particle crosses the spine curve.

Consider now the separatrices of the two-dimensional field. By setting $d z=0, E_{1}=E, E_{2}=0$, and $\kappa=1$ we obtain two separatrix planes, $x=0$ and $y=-a_{1} x / 2$. These intersect at the neutral line and define a narrow $\mathrm{X}$-point of angle
$2 / a_{1}=2 \alpha \lambda \eta / E$. The three-dimensional solution shows that although the plane of the current sheet survives the transition from two to three dimensions, the advection plane "collapses" into the spine curve (see also Lau \& Finn 1990).

\section{SUMMARY}

The development of magnetic-merging theory over the last 30 years has undoubtedly been hampered by the absence of exact reconnection solutions and by the difficulty of performing two- and three-dimensional reconnection simulations at realistic plasma resistivities (see, e.g., Biskamp 1994). We have shown, however, that the two-dimensional analysis of Craig \& Henton (1995) — which provides the only exact, analytic, nonlinear reconnection solution yet known-can be naturally extended to three dimensions. In this case, reconnection involves fan surfaces and spine curves as opposed to intersecting X-point planes. The present solution shows explicitly that advection across the spine curve leads to a global current sheet aligned to the fan-in other words, to "fan current reconnection."

The solution also suggests a further possibility, that reconnection may be driven by advection through the fan generating currents aligned to the spine. In fact, exact solutions for "spine current reconnection" can be obtained by a natural extension of the present analysis (Craig \& Fabling 1996).

It is a pleasure to acknowledge helpful discussions with Alfred Sneyd and the contribution of the Fly plotting code from Sandy McClymont. This work was partially funded by a FORST research grant.

\section{REFERENCES}

Besser, B. P., Biernat, H. K., \& Rijnbeek, R. P. 1991, J. Atmos. Terr. Phys., 53 1081

Biskamp, D. 1994, Phys. Rep., 4, 237

Craig, I. J. D., \& Fabling, R. B. 1996, ApJ, in press

Craig, I. J. D., \& Henton, S. M. 1995, ApJ, 450, 280

Jardine, M., Allen, H. R., Grundy, R. E., \& Priest, E. R. 1992, J. Geophys. Res., 97, 4199
Lau, Y. T. \& Finn, J. M. 1990, ApJ, 350, 672

Phan, T. D., \& Sonnerup, B. U. Ö. 1990, J. Plasma Phys., 44, 525 Priest, E. R., \& Titov, V. S. 1995, Phil. Trans. R. Soc. London A, in press Sonnerup, B. U. Ö., \& Priest, E. R. 1975, J. Plasma Phys., 14, 283 
